\title{
Measuring the performance of a distributed interference management scheme in a LTE-based HetNet deployment
}

\author{
Oriol Font-Bach, Nikolaos Bartzoudis and Miquel Payaró \\ Centre Tecnològic de Telecomunicacions de Catalunya (CTTC) \\ Castelldefels, Spain \\ Email: \{ofont, nbartzoudis, mpayaro\}@cttc.cat
}

\author{
Antonio Pascual-Iserte \\ Dept. Signal Theory and Communications \\ Universitat Politècnica de Catalunya (UPC) \\ Barcelona, Spain \\ Email: antonio.pascual@upc.edu
}

\begin{abstract}
This paper presents the experimental evaluation of an interference-mitigation scheme tailored for heterogeneous multi-cell LTE-based systems. The real-time FPGA-based system implementation was evaluated under realistic operating conditions using the GEDOMIS ${ }^{\circledR}$ testbed. An intensive measurement campaign was conducted to assess the performance-gains of the proposed scheme, considering mobile channels and various levels of noise and interference.
\end{abstract}

\section{INTRODUCTION}

The coexistence of macro cellular networks with small cell deployments will play an important role in the forthcoming generation of wireless communications, usually denoted as $5 \mathrm{G}$. One of the main goals in such heterogeneous network (HetNet) topologies is the reuse of the available radio frequency (RF) spectrum, which is not optimally exploited in current cellular radio access networks. This becomes increasingly important considering the growing number of wireless access users and the emerging trends in mobile computing, with an everincreasing requirement for high data-rate communications. The opportunistic reuse of RF spectrum in heterogeneous multi-cell network deployments unavoidably leads to in-band interference. Hence, an inherent operational requirement of HetNets is the implementation of efficient interference management schemes. A typical real-life case is encountered in LTEbased femtocell deployments, where interference management is a de-facto prerequisite in the joint design of the physical (PHY) and medium access control (MAC) layers of network infrastructure and user equipment (UE).

As far as the PHY-layer is concerned, the inclusion of an interference management scheme typically requires a rather bit-intensive interference-detection mechanism at UE-level, specific inter-cell signalling among the different nodes, and adaptive subcarrier allocation at the femto base stations (BSs) to enable generation of non-interfering radio frames. The performance of the interference management algorithms depends on a series of factors such as the propagation channel, the UE mobility, the power of the interfering signal and the noise level. Thus the best practice for validating them is to design, implement and test them in a real-time hardware setup that generates and processes signals subject to real-life impairments and hardware-constraints.

This paper presents measurement campaign results of an interference management scheme that was developed for a LTEbased macrocell/femtocell coexistence operating scenario. The baseband building blocks of all the entities were implemented in field programmable gate array (FPGA) devices using a custom hardware description language (HDL) design approach [1]. The real-time FPGA implementation encapsulated advanced parallel processing techniques and a hardware-aware optimization of the arithmetic operations. In order to achieve realistic validation conditions, the resulting real-time implementation was tested and evaluated with the help of the GEDOMIS ${ }^{\circledR}$ testbed [2].

\section{CONSIDERED INTERFERENCE-MANAGEMENT SCHEME}

In the considered scenario (see Fig. 1) the macrocell represents the primary communication, while the femtocell applies an opportunistic transmission scheme based on frequencyreuse. Both systems are simultaneously making use of the same operating RF band and downlink (DL) signal bandwidth (BW). The adopted interference management strategy is based on a self-organized physical resource block (PRB) allocation scheme for the femto BS; if the macro UE detects interference in the DL signal, it notifies its associated BS (through a dedicated feedback link) which, in turn, requests the femto BS to adapt its transmission in order to mitigate the interference. In this paper, we consider a simplified version of this interference management scheme that comprises a single pair of macro BS and UE as well as its femto counterparts (pointto-point DL communications). The considered PHY-layer is based on the LTE standard (rel. 9) [3] with the specifications listed in Table I. While the macro transmission uses the whole bandwidth (i.e., $20 \mathrm{MHz}$ ), two $10 \mathrm{MHz}$ bands are defined for the opportunistic femto communication. The femto BS uses those subbands for its opportunistic transmission where no interference is detected by the macro UE (i.e., there are 4 predefined femto PRB-allocation cases, ranging from a whole BW transmission to no-transmission state).

The interference detection uses a triggering-threshold technique, which takes into account the amount of received inter- 


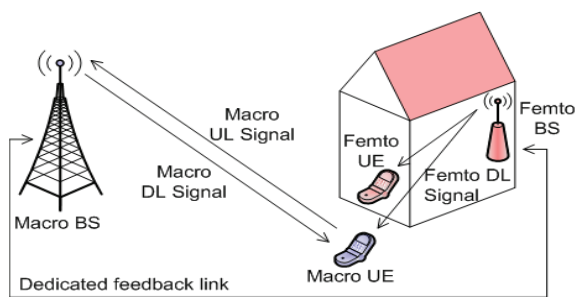

Fig. 1: Considered LTE-based macrocell/femtocell scenario.

TABLE I: Main PHY-layer specifications of the system.

\begin{tabular}{c|c} 
Parameter & Value \\
\hline \hline Antenna scheme & SISO \\
\hline RF operating band $(\mathrm{GHz})$ & 2.6 \\
\hline Channel BW $(\mathrm{MHz}) \mid$ FFT size & $20 \mid 2048$ \\
\hline Baseband | ADC sampling freq. (MHz) & $30.72 \mid 61.44$ \\
\hline Cyclic prefix (samples) & $512(1 / 4$ symbol) \\
\hline Duplex mode & FDD (DL) \\
\hline Channel models & ITU Pedestrian B \\
& 3GPP Extended Pedestrian A \\
\hline
\end{tabular}

ference and also the degradation of the values of the crosscorrelation utilized by the synchronization block at the receiver. The defined threshold-value aims at ensuring a baseline macro UE performance: the probability that the uncoded biterror-rate (BER) is below $10^{-2}$ must be above 0.8 [1].

\section{OVERVIEW OF THE Related Literature}

High-level programming languages and computer-based simulations provide a cost-effective, rapid and flexible approach to model and verify novel interference-management algorithms for HetNet systems [4], [5], [6]. On the other hand, when such algorithms are implemented in real-time DSP prototyping boards and RF transceivers, they take into account real-life operating constraints, implementation losses, component imperfections and signal impairments. Real-time implementations also allow to assess the performance of the PHY-layer under different functional scenarios that take into account the dynamic behaviour of continuous operation and are subject to the effects of realistic propagations channels (simulation analysis is typically limited to short-length system validation).

Another popular approach for validating interferencemanagement algorithms is to interface the baseband part of BSs or UEs realized in computer-based simulations with commercial-off-the-shelf (COTS) equipment. An indicative example is provided in [7], where an interference alignment technique for MIMO-OFDM systems is validated combining over-the-air transmissions with a Matlab-developed PHY-layer. Similarly, the gains of coordinated multi-point (CoMP) in a LTE system are evaluated in [8] through computer-simulations that uses field-trial data-captures. However, when considering this rapid prototyping approach, the computing limitations of the general purpose computer simulating the PHY-layer make it difficult to account for realistic UE mobility, large BWs or run-time adaptivity. In addition, there are setups that

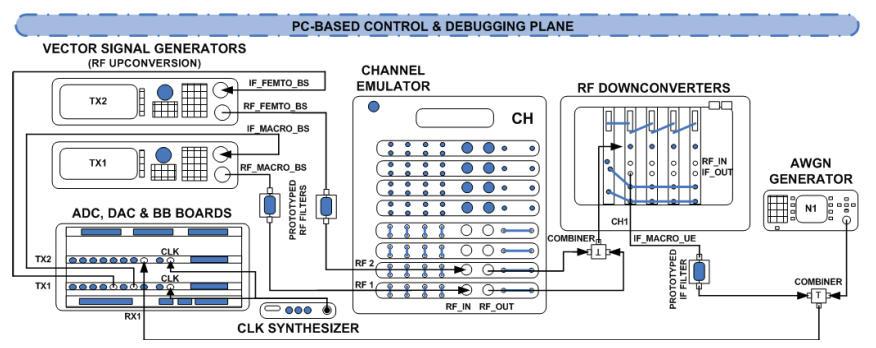

Fig. 2: Utilized GEDOMIS testbed setup.

use COTS end-products that include the baseband modems as well, allowing a full real-time operation of the system. For instance, the authors in [9] analyse the performance gains of an uplink antenna selection technique in a LTE-FDD femtocell system, where a channel emulator provides mobility conditions. Similarly, in [10] it is measured and modelled the level of interference caused by indoor LTE-based femtocells through an exhaustive measurement campaign, including UE mobility and a wide range of different femto-BS locations. Nevertheless, such COTS-based setups can only serve the goals of performance benchmarking as usually the PHY layer of end-products is not accessible for further development. On top of that, in all previous cases it is neither considered nor measured the effect of femto-interference onto neighbouring macro UEs.

In recent trends of PHY-layer prototyping software defined radio (SDR) techniques have been widely adopted due to its programming flexibility, short development time and increasing performance enhancements of modern (embedded) microprocessors. However, the encountered cases in the literature typically report limited laboratory validation results of the proposed cognitive radio functionalities [11], [12]. Another common situation is that of partial system implementations (i.e., behavioural HDL simulations or FPGA-synthesis results), such as the one presented in [13], where it is described the design and real-time implementation of a distributed beamforming algorithm for interference mitigation in LTE systems. Custom real-time implementations of the PHY-layer allow to overcome all the limitations described before at the expense of a rather longer development time.

\section{IMPLEMENTATION OF THE CONSIDERED SCENARIO USING THE GEDOMIS TESTBED}

The PHY-layer of the two BSs and UEs of the target interference management scenario was prototyped using the FPGA-based development boards of the GEDOMIS testbed [1]. The baseband boards also include high-end multichannel signal conversion circuitry. Two Agilent ESG4438C vector signal generators (VSGs) were used for the RF up-conversion. At the receiver's end, the RF downconversion was carried out with the help of a 4-channel Mercury Computer Systems Echotek Series RF 3000T Tuner. The Elektrobit Propsim C8 real-time multi-channel emulator provides the ability to test and validate both standard and custom channels that may include mobility of the UEs. 


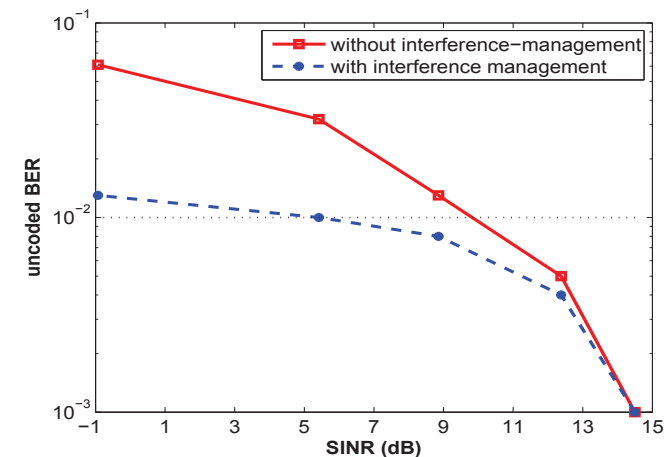

Fig. 3: Macro UE performance under a static PB channel.

In order to deploy the described macrocell/femtocell interference-mitigation scenario, GEDOMIS was configured as shown in Fig. 2. The specific interference, noise and channel propagation conditions were achieved by configuring the channel emulator to provide the real-time response of two uncorrelated SISO channels: (1) a channel implementing either the ITU Pedestrian B (PB) or 3GPP Extended Pedestrian A (EPA) models (considering both static and mobile channel conditions) was applied to the RF signal generated by the macro BS and (2) a custom channel model that was applied to the RF signal generated by the femto BS, in order to produce one of the four possible interference scenarios. This was made feasible by defining two custom channel models, one with a response similar to a high-pass filter and another with a response similar to a low-pass filter (i.e., filtering one of the two $10 \mathrm{MHz}$ bands of the signal generated by the femto BS). Hence, a static channel impulse response was considered for the femto DL signal, which results in a constant-power interference to the macro UE. The two RF outputs of the channel emulator corresponding to the macro BS and the interfering signal were combined and fed to the RF downconverter equipment. An Applied Instruments NS-3 RF Noise Source device was used to provide a flat additive white Gaussian noise (AWGN) signal, which was combined with the IF output of the downconverters, provided the DL signal received by the macro UE. Additionally, the settings of the channel emulator could be adjusted at run-time to provide a precise interference level. The gain level of different amplifiers and attenuators encountered in the entire signal path of the testbed were exhaustively tested for every $1 \mathrm{~dB}$ attenuation step within the desired Signal-to-Interference-plus-Noise ratio (SINR) range, which was set between 15 and $-10 \mathrm{~dB}$ according to the pathloss model for suburban deployment of LTE femtocells defined by the 3GPP [14]. Finally, the precise time-shift (i.e., in samples) of the DL macro and interference signals was fully controlled using a user-programmable register located at the FPGA implementation of the two BSs.

\section{Testing Methodology AND EXPERIMENTAL RESUlts}

Data was captured at the outputs of the AGC stage of the macro UE in order to obtain the SINR of the received DL

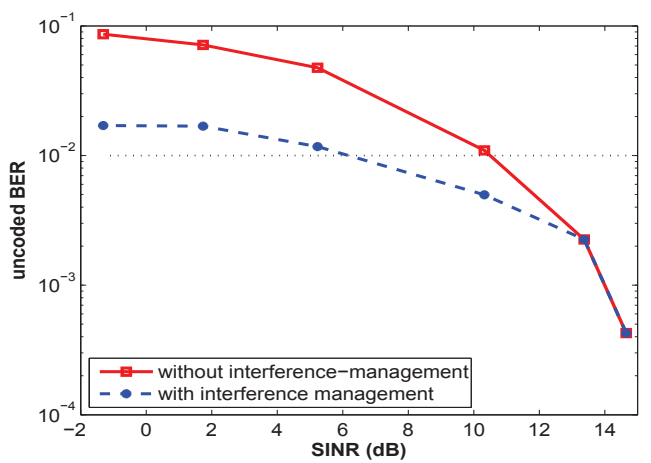

Fig. 4: Macro UE performance under a static EPA channel.

signal. This was achieved by buffering the FPGA-signals of interest to a large on-board random access memory (RAM). The data was then retrieved and post-processed in Matlab. On top of that, the calculation of the instantaneous (i.e., per frame) uncoded BER at the macro UE was implemented in dedicated FPGA-logic. For each of the eight considered SINR values and for each channel realization two complete postAGC frames (i.e., more than 600000 I/Q 32-bit samples) and 16384 BER values (i.e., each corresponding to a unique $10 \mathrm{~ms}$ radio frame) were captured. Matlab scripts were then employed to calculate the average SINR and BER values. It is important to underline that a single time-variant channel realization (i.e., using a unique channel-distribution seed) was implemented for each considered channel model and mobile speed. Whereas the resulting performance analysis was not exhaustive (due to the limited amount of data that was processed), it provides a significant insight on the effectiveness of the proposed interference-management scheme under realistic operating conditions. A complete validation would require to repeat the data-capturing and post-processing procedures for at least 100 different realizations of each considered mobile channel and speeds.

Fig. 3 shows the performance-gains leveraged by the proposed interference-management scheme when considering a single static PB channel realization for different SINR values at the macro UE receiver. A gain of approximately $5 \mathrm{~dB}$ is obtained in respect to the target baseline macro UE performance (i.e., $10^{-2}$ ). Almost identical results are obtained when repeating the experiment using a static EPA channel realization, as it can be observed in Fig. 4.

Mobility was then progressively introduced in the experimental setup, in order to analyse the effects of a time-varying channel response on the interference-mitigation scheme. A very low user mobility of $0.2 \mathrm{~km} / \mathrm{h}$ was first applied to both single PB and EPA channel realizations, as shown in Fig. 5 and Fig. 6 respectively. A gain about $2 \mathrm{~dB}$ was still achieved around the defined threshold performance-level. As expected the effectiveness of the proposed interference-detection technique was degraded in the presence of mobile channels. Finally, a standard pedestrian mobile speed of $3 \mathrm{~km} / \mathrm{h}$ was applied to a unique realization of both PB (Fig. 7) and EPA 


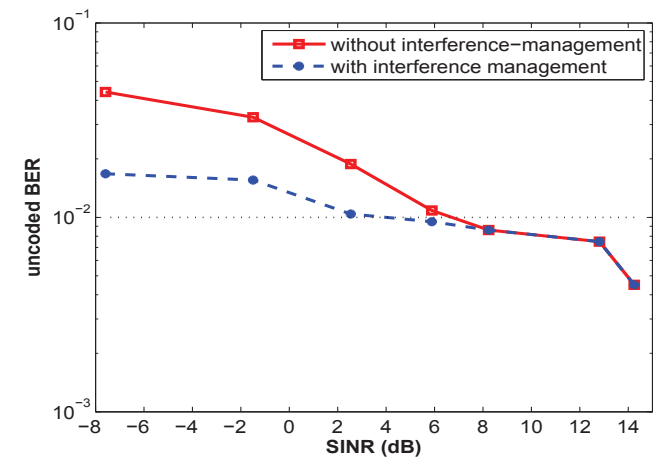

Fig. 5: Macro UE performance in a low-speed PB channel.

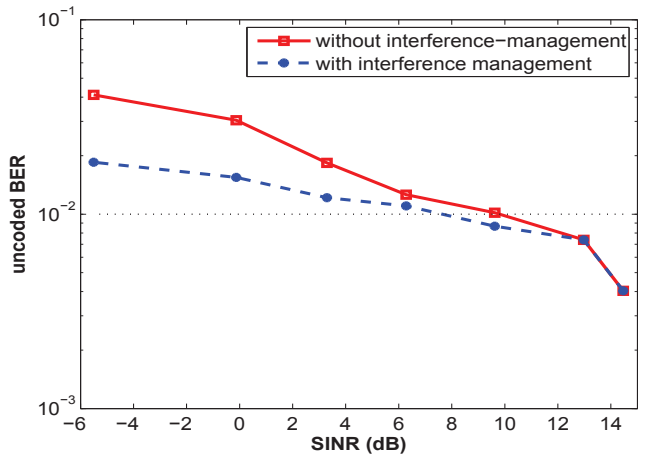

Fig. 6: Macro UE performance in a low-speed EPA channel.

(Fig. 8) channels. As it is anticipated, under higher mobility conditions the achieved gain of the interference-mitigation scheme has dropped in the range of $1.2 \mathrm{~dB}$, thus minimally extending the SINR-range where the desired baseline BER performance is met.

\section{CONCLUSions}

This paper presented the prototyping and experimental evaluation of an interference-mitigation scheme in a LTEbased macrocell/femtocell scenario. The proof-of-concept was deployed using the GEDOMIS testbed to obtain close to real-life operating conditions through a complex laboratory setup, which includes a real-time channel emulator. Hence, the presented performance analysis takes into account the realistic signal impairments of the considered mobile channels and those introduced by the entire hardware setup.

\section{ACKNOWLEDGMENT}

This work was partially supported by: the Spanish Government under projects TEC2011-29006-C03-01 (GRE3N-PHY) and TEC2011-29006-C0302 (GRE3N-LINK-MAC); and the European Commission under project NEWCOM\# (GA 318306).

\section{REFERENCES}

[1] O. Font-Bach, N. Bartzoudis, A. Pascual-Iserte, M. Payaró, L. Blanco, D. López Bueno and M. Molina, "Interference management in LTE-based HetNets: practical approach," in Transactions on Emerging Telecommunications Technologies, 2014 (available on-line, DOI: 10.1002/ett.2833).

[2] GEDOMIS ${ }^{\circledR(}$ testbed, http://engineering.cttc.es/gedomis.

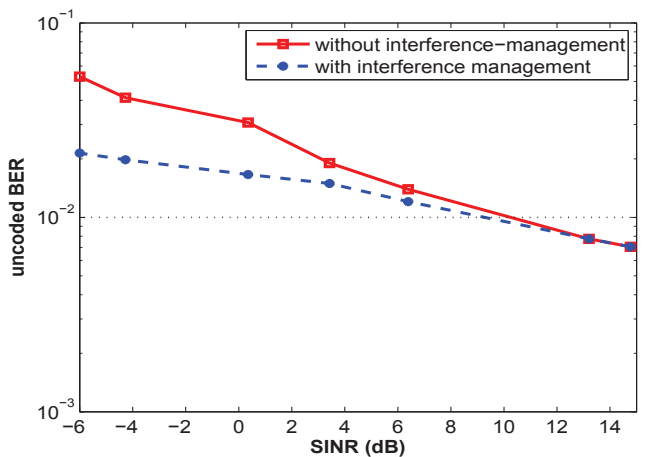

Fig. 7: Macro UE performance in a $3 \mathrm{~km} / \mathrm{h}$ PB channel.

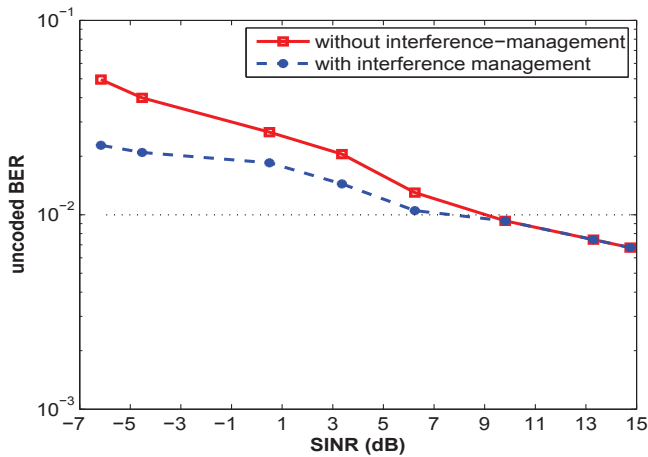

Fig. 8: Macro UE performance in a $3 \mathrm{~km} / \mathrm{h}$ EPA channel.

[3] 3GPP LTE Rel. 9, "Evolved Universal Terrestrial Radio Access (E-UTRA); Physical Channels and Modulation", 3GPP TS 36.211, 2010

[4] F. Mhiri, K. Sethom, and R. Bouallegue, "A survey on interference management techniques in femtocell self-organizing networks," Journal of Network and Computer Applications, 2012.

[5] A. Latrach, R. Nasri and S. Affes, "Downlink Interference Cancellation Strategy for Shared-Spectrum LTE HetNet," in Proc. International Wireless Communications and Mobile Computing Conference (IWCMC), Jul 2013.

[6] J.-J. Abularach Arnez, L. da Silva Mello and C. Rodriguez R., "Simulation of LTE Femtocell Interference Scenarios for Cognitive Radio Implementations," in Proc. SBMO/IEEE MTT-S International Microwave Optoelectronics Conference (IMOC), Aug 2013.

[7] J. A. García-Naya, L. Castedo, O. González, D. Ramírez, and I. Santamaria, "Experimental Evaluation of Interference Alignment under Imperfect Channel State Information," in Proc. European Signal Processing Conference (EUSIPCO), Aug. 2011.

[8] R. Irmer, H. Droste, P. Marsch, M. Grieger, G. F. and S. Brueck, H.-P. Mayer, L. Thiele, and V. Jungnickel, "Coordinated Multipoint: Concepts, Performance, and Field Trial Results," IEEE Communications Magazine, vol. 47, no. 2, pp. 102-111, Feb. 2011.

[9] J. Soler-Garrido and W. H. Chin, "Performance of an LTE Femtocell Base Station Employing Uplink Antenna Selection," in Proc. Wireless Advanced (WiAd), Jun. 2011.

[10] D.-M. Rose, T. Jansen and T. Kürner, "Indoor to Outdoor Propagation - Measuring and Modeling of Femto Cells in LTE Networks at 800 and $2600 \mathrm{MHz}$," in Proc. IEEE Global Communications Conference (GLOBECOM) Workshops, Dec 2011.

[11] L. Zhao, S. Chen, and X. Gan, "From Reconfigurable SDR to Cognitive Femto-Cell: A Practical Platform," in Proc. International Conference on Wireless Communications and Signal Processing (WCSP), Nov. 2011.

[12] D.-R. Hsieh, D.-J. Huang, J.-Y. Hsu, C.-Y. Kao, M.-C. Lin, C.-N. Liu and P. Ting, "Baseband Design and Software-Defined-Radio Implementation for LTE Femtocell," in Proc. Asian Control Conference (ASCC), Jun 2013.

[13] A. Krdu, Y. Lebrun, U. Ahmad, S. Pollin, P. Agrawal and M. Li, "Beamforming for Interference Mitigation and its Implementation on an SDR Baseband Processor," in Proc. IEEE Signal Processing Systems (SiPS) Workshops, Oct 2011.

[14] T. G. P. P. (3GPP), "Simulation assumptions and parameters for FDD HeNB R requirements, 3GPP TSG RAN WG4 R4-092042, 2009. 